\title{
Entanglement in a Maxwell theory coupled to a non-relativistic particle
}

\section{Filiberto Ares, ${ }^{a}$ Amilcar R. de Queiroz ${ }^{b}$ and Marcia R. Tenser ${ }^{b, c}$}

${ }^{a}$ International Institute of Physics, Universidade Federal do Rio Grande do Norte, C.P. 1613, 59078-970, Natal, RN, Brazil

${ }^{b}$ Instituto de Física, Universidade de Brasília, C.P. 04455, 70919-970, Brasília, DF, Brazil

${ }^{c}$ Instituto de Física, Universidade de São Paulo, C.P. 66318, 05315-970, São Paulo, SP, Brazil

E-mail: fares@iip.ufrn.br, amilcarq@gmail.com, marciatenser@gmail.com

ABSTRACT: We consider electromagnetism in a cylindrical manifold coupled to a nonrelativistic charged point-particle. Through the relation between this theory and the Landau model on a torus, we study the entanglement between the particle and the electromagnetic field. In particular, we compute the entanglement entropy in the ground state, which is degenerate, obtaining how it varies in the degeneracy subspace.

KEYwords: Field Theories in Lower Dimensions, Gauge Symmetry, Integrable Field Theories, Nonperturbative Effects

ARXIV EPRINT: 1911.11264 


\section{Contents}

1 Introduction 1

2 Electromagnetism on a space-time cylinder and the Landau model on a torus

2.1 Electromagnetism coupled to a charged point particle 3

2.2 Landau problem on a torus 5

$\begin{array}{llr}3 & \text { Entanglement entropy } & \mathbf{7}\end{array}$

$\begin{array}{ll}3.1 & \text { Entanglement entropy for a } V_{\theta} \text {-invariant state } \\ \end{array}$

4 Conclusions $\quad 15$

$\begin{array}{ll}\text { A Green's function } & 16\end{array}$

\section{Introduction}

Being the characteristic trait of quantum mechanics [1], entanglement has been revealed as fundamental in many fields and phenomena ranging from quantum information $[2,3]$, and condensed matter [4-6] to black hole physics [7]. One of the standard quantities employed to characterise entanglement is entanglement entropy (EE). In order to define it, we must partition the system into two parts such that its Hilbert space is the tensor product of the Hilbert space of the partitions. Then the EE measures the degree of entanglement between the two subsystems [8].

In field theories, one possibility is to divide the real space into several regions. This was originally done in a scalar field theory $[9,10]$ motivated by the Bekenstein-Hawking formula for a black hole. Later the analysis of spatial entanglement was extended to conformal field theories [11, 12] that the Ryu-Takanayagi formula [13] connects with gravity [14] via the holographic principle. Spatial EE in massive field theories has also been well studied, see for example [15-20]. One can also consider the entanglement between other partitions of the Hilbert space that are not spatial ones, as, for example, between right and left moving excitations [21-23] or between winding modes [24].

When the field theory presents gauge symmetry, spatial partitions are subtle: it is not possible to make them and still preserve gauge invariance. This difficulty is due to the fact that gauge theories contain non local degrees of freedom such as Wilson loops. Hence, when a spatial partition is made, these loops are necessarily broken. So we are left with an arbitrary choice (and therefore an ambiguity) of deciding to which of the subregions the broken degrees of freedom belong. Many aspects of this problem have been addressed in the literature since the work [25]. It has been discussed in the context of lattice 
gauge theory giving rise to different prescriptions for computing EE [26-31]. In the continuum, one possibility is to calculate EE using the replica trick after extending the Hilbert space in a particular way [32-34]. Without resorting to the replica trick, some alternative approaches have also been considered. For example, in [35], EE in 2+1 dimensions was studied employing gauge-invariant variables. In [36], the zoo of prescriptions for computing EE was unified using an algebraic approach, defining it in terms of a subalgebra of gaugeinvariant operators associated to each subregion. Another algebraic framework based on the Gel'fand-Naimark-Segal construction was suggested formerly in $[37,38]$ in order to treat systems of identical particles. This method was also applied to analyse the ambiguities of EE in systems with gauge symmetries [39, 40]. Recently in [41] the authors proposed that a proper measure for spatial entanglement in a Maxwell theory is mutual information. As one of the motivations to use this quantity instead of the bare entanglement entropy, they argue that it resolves the aforementioned ambiguities. This idea had been presented previously by the same authors in the context of systems with global symmetries in [42].

The works mentioned in the previous paragraph concern pure gauge theories, without coupling to matter. By including matter, one may not only study spatial entanglement [43] but also the entanglement between the gauge field and the matter sectors. The present paper is dedicated to the latter situation. This problem has also been investigated recently in [44] where the entanglement between a quantum harmonic oscillator and a quantized electromagnetic field was analysed.

Here we consider a non-relativistic particle coupled to an Abelian Yang-Mills (YM) theory in $1+1$ dimensions with compactified spatial coordinate, i.e., space-time is a cylinder $\mathbb{R} \times S^{1}$. In order to compute the EE between the particle and the field, we map the theory to a quantum mechanical system consisting of a charged particle moving on a torus with a uniform transverse magnetic flux. This is the Landau problem [45] on a torus [46]. In fact, as shown in [47], the field dynamics of a pure YM theory defined on a cylinder can be reduced to that of a free particle moving along the gauge group manifold. This means that we can reduce the quantum field theory problem to a quantum mechanical one. For simplicity, here we restrict to the Abelian case, in which the field theory is mapped to a particle moving on a circle [48]. From the field theory point of view, the only gauge-invariant observable is the Wilson loop along $S^{1}$. If we were to partition this circle to compute some kind of spatial entanglement, then we would break the gauge invariance and we would need to apply the techniques cited above. The fact that there is only one gauge invariant observable in the field theory implies that there is a single degree of freedom associated to the gauge field in the quantum mechanical theory. Thus if we do not consider matter, we have only one degree of freedom (a particle moving on a circle) and, therefore, it is impossible to make any partitions in this setup. Including a non-relativistic particle adds another degree of freedom (the particle moves now on a torus) and the possibility of making a partition. Hence the goal of this work is to understand the entanglement between the degree of freedom associated to the gauge field and that corresponding to the non-relativistic particle.

The paper is organised as follows: in the next section, we show the equivalence between electromagnetism on a space-time cylinder coupled to a non-relativistic charged point particle and the Landau problem on a torus. In section 2.2 , we obtain the solution of the 
Schrödinger equation of the latter, finding that the ground state is degenerate. In section 3 , we study the entanglement entropy in this degeneracy subspace. This is equivalent to measuring the entanglement between the particle and the electromagnetic field in the ground state of the field theory. In particular, we perform both an analytical and a numerical analysis of this quantity. We find that the reduced density matrix in the degeneracy subspace can be approximated by that of a two-level system. This observation allows us to obtain an analytical expression for the entanglement entropy in this subspace whose accuracy is checked numerically. We also study the entanglement entropy in the state that is invariant under the symmetry transformation associated to the degeneracy subspace. Finally, in section 4, we present the conclusions and outlooks. We include an appendix A where we compute the Green's function of the electric potential in the Maxwell theory on the space-time cylinder.

\section{Electromagnetism on a space-time cylinder and the Landau model on a torus}

In this section, we introduce the model to be discussed later and fix the notation. The model is electromagnetism on a space-time cylinder coupled to a non-relativistic charged point particle. We review its relation with the Landau model on a torus, that is, a charged particle moving on a torus subject to a transverse magnetic field.

\subsection{Electromagnetism coupled to a charged point particle}

We consider a space-time cylinder with coordinates $\mathbf{s}=\left(s^{0}, s^{1}\right) \equiv(t, s)$, where $t \in \mathbb{R}$ and $s \in[0,2 \pi R)$. If $\tau$ is the proper time of the point particle, with electric charge $q$ and mass $m$, then its classical trajectory can be parametrized as $\mathbf{r}(\tau)=\left(r^{0}(\tau), r^{1}(\tau)\right) \equiv(t(\tau), r(\tau))$. The electromagnetic field is described by $F_{\mu \nu}=\partial_{\mu} A_{\nu}-\partial_{\nu} A_{\mu}, \mu, \nu=0,1$. The electric field is $E=F^{01}$. The field equations are $\partial_{\nu} F^{\mu \nu}=J^{\mu}$, where ${ }^{1}$

$$
J^{\mu}(t, s)=q \int \mathrm{d} \tau \delta^{(2)}(\mathbf{s}-\mathbf{r}(\tau)) \frac{\mathrm{d} r^{\mu}(\tau)}{\mathrm{d} \tau}
$$

If we align the proper time $\tau$ with the time coordinate of the particle, so that $t=\tau$, then we have $J^{0}=q \delta(s-r(t)) \equiv \rho$ and $J^{1}=q \delta(s-r(t)) \dot{r} \equiv j$. Therefore, in local coordinates, the field equations read

$$
\begin{aligned}
& \partial_{s} E=-\partial_{s} \partial_{t} A_{1}+\partial_{s}^{2} A_{0}=\rho, \\
& \partial_{t} E=-\partial_{t}^{2} A_{1}+\partial_{t} \partial_{s} A_{0}=-j .
\end{aligned}
$$

For simplicity, we consider a non-relativistic charged point particle. Its equation of motion reads $^{2} m \ddot{r}=q E$. From now on, we set $m=1$.

The above equations of motion are obtained from the Lagrangian

$$
L=L_{\mathrm{M}}+L_{\mathrm{EM}}=\frac{\dot{r}^{2}}{2}+\int \mathrm{d} s\left(\frac{\left(\partial_{t} A_{1}-\partial_{s} A_{0}\right)^{2}}{2}+A_{0} \rho+A_{1} j\right) .
$$

\footnotetext{
${ }^{1} \delta^{(2)}(\mathbf{s}-\mathbf{r}(\tau))=\delta\left(s^{0}-r^{0}(\tau)\right) \delta\left(s^{1}-r^{1}(\tau)\right)$.

${ }^{2} m \ddot{r}^{\mu}=q F^{\mu \nu} \dot{r}_{\nu}$.
} 
In the Coulomb gauge, $\partial_{s} A_{1}=0$, the Gauss law (2.2) becomes $\partial_{s}^{2} A_{0}=\rho$. Therefore, $A_{0}$ is not dynamical and its only role is enforcing the Gauss law. This constraint can be readily solved as

$$
A_{0}(s)=\int \mathrm{d} s^{\prime} G\left(s, s^{\prime}\right) \rho\left(s^{\prime}\right),
$$

where $G\left(s, s^{\prime}\right)$ is the Green's function of the operator $\partial_{s}^{2}$. Generically, finding this Green's function depends on the boundary conditions. We will take them to be periodic, such that $A_{0}(s+2 \pi R)=A_{0}(s), \rho(s+2 \pi R)=\rho(s)$, and, therefore, $G\left(s+2 \pi R, s^{\prime}\right)=G\left(s, s^{\prime}+2 \pi R\right)=$ $G\left(s, s^{\prime}\right)$. In appendix A, we describe in detail the calculation of $G\left(s, s^{\prime}\right)$. We find

$$
G\left(s, s^{\prime}\right)=-\frac{\left([s]-\left[s^{\prime}\right]\right)^{2}}{4 \pi R}+\frac{\left|[s]-\left[s^{\prime}\right]\right|}{2}-\frac{\pi R}{6},
$$

where the notation $[\bullet]$ stands for $[\bullet] \equiv \bullet \bmod 2 \pi R$, and $s, s^{\prime} \in \mathbb{R}$.

In the Coulomb gauge, the field $A_{1}(t, s)$ does not depend on the spatial coordinate $s$, i.e., $A_{1}(t, s)=a(t)$. Moreover, gauge invariance implies that $a(t)$ is valued on a circle of length $\frac{1}{e R}$. In fact, consider a gauge transformation $g=\mathrm{e}^{\mathrm{i} e \Lambda(t, s)}$, where $e$ denotes the elementary electric charge, that winds around the spatial dimension. In order to be a single-valued transformation, $\Lambda(t, s)$ must satisfy

$$
\Lambda(t, s=2 \pi R)=\Lambda(t, s=0)+\frac{2 \pi n}{e}, \quad \text { for some } n \in \mathbb{Z} .
$$

A possible solution is $\Lambda(t, s)=\frac{n s}{e R}$. In this case, the gauge field transforms as

$$
A_{1}(t, s) \mapsto A_{1}(t, s)+\partial_{s} \Lambda(t, s)=A_{1}(t, s)+\frac{n}{e R} .
$$

The equivalence of configurations of the field related by gauge transformations implies that we can restrict $a(t)$ to $0 \leq a(t)<\frac{1}{e R}$.

Now going back to the Lagrangian, the Coulomb gauge allows us to rewrite $L_{\mathrm{EM}}$ as

$$
L_{\mathrm{EM}}=\int_{0}^{2 \pi R} \mathrm{~d} s\left(\frac{\dot{a}^{2}}{2}+\frac{A_{0} \rho}{2}+j a\right),
$$

where we have used that $\partial_{s}^{2} A_{0}=\rho$ and the periodicity of $A_{0}(s)$. Applying (2.5), we then obtain

$$
L_{\mathrm{EM}}=\int \mathrm{d} s\left(\frac{\dot{a}^{2}}{2}+j a\right)+\frac{1}{2} \int \mathrm{d} s \mathrm{~d} s^{\prime} \rho(s) G\left(s, s^{\prime}\right) \rho\left(s^{\prime}\right) .
$$

Taking into account that, according to (2.6), $G(r(t), r(t))=-\pi R / 6$, the Lagrangian (2.4) can be written as

$$
L=\frac{\dot{r}^{2}}{2}+\frac{\dot{a}^{2}}{2} 2 \pi R+q \dot{r} a-\frac{q^{2} \pi R}{12} .
$$

The term $-q^{2} \pi R / 12$ can be dropped since it does not affect the equations of motion. After completing the square, we finally arrive at

$$
L=\frac{\dot{a}^{2}}{2} 2 \pi R+\frac{1}{2}(\dot{r}+q a)^{2}-\frac{q^{2}}{2} a^{2} .
$$


From now on, we define the elementary electric charge as $e=2 \pi$ and write the charge of the particle as $q=-e \theta=-2 \pi \theta$. We also choose the specific value $R=\frac{1}{2 \pi}$, such that the spatial direction has unit length. Observe that, under these considerations, the gauge field $a(t)$ is also valued on a circle of length one. Moreover, $a$ may be replaced by $x$ and the position of the particle $r$ by $y$. Therefore, $x \in[0,1), y \in[0,1)$ and the Hamiltonian corresponding to the Lagrangian (2.12) reads

$$
H=\frac{p_{x}^{2}}{2}+\frac{1}{2}\left(p_{y}+2 \pi \theta x\right)^{2},
$$

where

$$
p_{y}=\dot{y}-2 \pi \theta x \quad \text { and } \quad p_{x}=\dot{x} .
$$

Since we are interested in studying the entanglement between the electromagnetic and the matter sector, in the following sections we will consider the quantum version of the Hamiltonian (2.13). It can be straightforwardly found through canonical quantization, i.e., by promoting $x, y$ and $p_{x}, p_{y}$ to operators acting on $L^{2}([0,1) \times[0,1))$ that satisfy the canonical commutation relations $\left[x, p_{x}\right]=\left[y, p_{y}\right]=\mathrm{i}$ and $\left[x, p_{y}\right]=\left[y, p_{x}\right]=0$.

\subsection{Landau problem on a torus}

The Hamiltonian (2.13) is also obtainable from the problem of a particle of charge 1 moving on a torus with local coordinates $0 \leq x, y<1$ in presence of a constant magnetic field $B_{z}=2 \pi \theta$ in the transverse direction. This is the famous Landau problem on a torus. In appendix $\mathrm{B}$ of [46] this model is studied in detail for the case $\theta=1$ (see also [48-50]). Note that the Hamiltonian (2.13) is written in the gauge $\mathcal{A}_{y}=2 \pi \theta x$ and $\mathcal{A}_{x}=0$, so that $B_{z}=\partial_{x} \mathcal{A}_{y}-\partial_{y} \mathcal{A}_{x}=2 \pi \theta$ (we denote the gauge field as $\mathcal{A}$ to avoid confusion with section 2.1). Moreover, we can define the momenta

$$
\pi_{x}=p_{x}+\mathcal{A}_{x}=p_{x}, \quad \text { and } \quad \pi_{y}=p_{y}+\mathcal{A}_{y}=p_{y}+2 \pi \theta x .
$$

There is a second set of translation operators that commute with the above momenta and, therefore, with the Hamiltonian (2.13). These translations are generated by

$$
v_{x}=p_{x}+2 \pi \theta y, \quad \text { and } \quad v_{y}=p_{y} .
$$

Although all the operators in (2.15) and (2.16) formally commute with the Hamiltonian (2.13) as differential operators, the system presents an anomaly and not all the translations generated by them are symmetries of the theory due to the boundary conditions $[49,51]$. The Hamiltonian (2.13) is self-adjoint if the wave functions satisfy

$$
\psi(0, y ; t)=\mathrm{e}^{2 \pi \mathrm{i} \theta y} \psi(1, y ; t), \quad \psi(x, 0 ; t)=\psi(x, 1 ; t) .
$$

Similar conditions apply to the first derivatives of the wave function. These boundary conditions define the domain of the Hamiltonian.

That fact implies that translational invariance is broken to the discrete cyclic subgroup $\mathbb{Z}_{\theta} \times \mathbb{Z}_{\theta}$, with $\theta \in \mathbb{Z}$ (see [50] for a comprehensive discussion). The infinitesimal translations 
generated by $v_{x}$ and $v_{y}$ do not respect the boundary conditions; that is, their images are states which in general do not fulfil (2.17) and, therefore, they are not in the domain of the Hamiltonian (2.13). As shown in [49], an operator is anomalous if it does not keep invariant the domain of the Hamiltonian. Only the discrete translations

$$
V_{x}(a)=\mathrm{e}^{\mathrm{i} \frac{a}{\theta} v_{x}}, \quad V_{y}(a)=\mathrm{e}^{\mathrm{i} \frac{a}{\theta} v_{y}}, \quad a=1, \ldots, \theta, \theta \in \mathbb{Z},
$$

map the domain of the Hamiltonian (2.13) into itself. As a consequence, they are the only translations that are actual symmetries of the theory.

First of all, let us see that $\theta$ has to be an integer. Recall that the length of the torus in each direction is 1 . Hence a full rotation around each direction of the torus is performed by

$$
V_{x} \equiv V_{x}(\theta)=\mathrm{e}^{\mathrm{i} v_{x}} \quad \text { and } \quad V_{y} \equiv V_{y}(\theta)=\mathrm{e}^{\mathrm{i} v_{y}} .
$$

Now, if one starts with a wave function satisfying (2.17) at the point $(x, y) \equiv(0,0)$ and transports it to $(1,1)$, there are two possible paths,

$$
\begin{array}{ll}
(0,0) \rightarrow(1,0) \rightarrow(1,1), & V_{y} V_{x} \psi(0,0)=V_{y} \psi(1,0)=\psi(1,1), \\
(0,0) \rightarrow(0,1) \rightarrow(1,1), & V_{x} V_{y} \psi(0,0)=V_{x} \psi(0,1)=\mathrm{e}^{2 \pi \mathrm{i} \theta} \psi(1,1) .
\end{array}
$$

Equivalently,

$$
V_{x}^{-1} V_{y}^{-1} V_{x} V_{y} \psi(0,0)=\mathrm{e}^{2 \pi \mathrm{i} \theta} \psi(0,0) .
$$

Notice that the charge $2 \pi \theta$ plays the role of a central charge for the translations on the torus. Moreover, since the final result at $(1,1)$ should be independent of the path, we obtain $\theta \in \mathbb{Z}$. This is electric charge quantization in the field theory and magnetic flux quantization in the Landau model.

The quantization of $\theta$ has also implications for the degeneracy of the Hamiltonian. In particular, the solutions to the stationary Schrödinger equation $H \psi_{k}(x, y)=E \psi_{k}(x, y)$ which are compatible with the boundary conditions (2.17) may be written as

$$
\psi_{k}(x, y)=\sum_{n \in \mathbb{Z}} \varphi_{n k}(x) \mathrm{e}^{2 \pi \mathrm{i} \theta\left(n+\frac{k}{\theta}\right) y}, \quad k=0, \ldots, \theta-1,
$$

where

$$
\varphi_{n k}(x)=f\left(x+n+\frac{k}{\theta}\right)
$$

with $f$ satisfying a harmonic oscillator equation with angular frequency $2 \pi \theta$. The energy levels are given by $E_{\lambda}=2 \pi \theta(\lambda+1 / 2)$ with $\lambda \in \mathbb{Z}^{*}$. Note that the wave functions are not defined for $\theta=0$, which would correspond to zero transverse magnetic field.

Observe that the stationary wave functions $\psi_{k}(x, y)$ are also eigenfunctions of the discrete translations (2.18) in the $y$ direction

$$
V_{y}(a) \psi_{k}(x, y)=\mathrm{e}^{2 \pi \mathrm{i} k a / \theta} \psi_{k}(x, y) .
$$

On the other hand, the discrete translations (2.18) in the $x$ direction do not leave $\psi_{k}(x, y)$ invariant but, defining $V_{\theta} \equiv V_{x}(1)$, they act as

$$
V_{\theta} \psi_{k}(x, y)=\psi_{k+1}(x, y) .
$$


As pointed out before, one can see that the rest of translations generated by the operators in (2.16) do not preserve the domain of $H$ and map $\psi_{k}(x, y)$ to a wave function that, in general, does not satisfy the boundary conditions (2.17).

In the following section, we will be interested in the ground state solutions, $\lambda=0$, where $\varphi_{n k}(x) \sim \mathrm{e}^{-\pi \theta\left(x+n+\frac{k}{\theta}\right)^{2}}$. Therefore, the ground state wave functions are given by

$$
\psi_{k}(x, y)=\mathcal{N} \sum_{n \in \mathbb{Z}} \mathrm{e}^{-\pi \theta\left(x+n+\frac{k}{\theta}\right)^{2}} \mathrm{e}^{2 \pi \mathrm{i} \theta\left(n+\frac{k}{\theta}\right) y}
$$

where $\mathcal{N}$ is a normalization constant. The wave function (2.27) can be rewritten using the Jacobi $\vartheta$ function,

$$
\vartheta_{3}(z \mid \tau)=\sum_{n \in \mathbb{Z}} \mathrm{e}^{\mathrm{i} \pi \tau n^{2}+2 \pi \mathrm{i} z n}
$$

in the form

$$
\psi_{k}(x, y)=\mathcal{N} \mathrm{e}^{2 \pi \mathrm{i} k y-\pi \theta(x+k / \theta)^{2}} \vartheta_{3}(\theta(\mathrm{i} x+y+\mathrm{i} k / \theta) \mid \mathrm{i} \theta)
$$

\section{Entanglement entropy}

In this section, we study the entanglement between the charged particle and the electromagnetic field in the ground state of the theory described by the Lagrangian (2.4). According to the analysis performed in section 2, this is equivalent to measuring the entanglement between the two degrees of freedom, $x$ and $y$, of the Landau model on a torus defined by the Hamiltonian (2.13). We shall compute the entanglement entropy in the ground state of the latter system. As we have seen in the previous section, the ground state is degenerate. We shall then analyse the entanglement entropy in the degeneracy subspace $\left\{\left|\psi_{k}\right\rangle\right\}_{k=0}^{\theta-1}$ where the vectors $\left|\psi_{k}\right\rangle$ are such that $\psi_{k}(x, y) \equiv\left\langle x, y \mid \psi_{k}\right\rangle$ with $\{|x, y\rangle\}$ being the coordinate basis and $\psi_{k}(x, y)$ being the ground state wave function given in $(2.27)$.

In order to define the entanglement entropy, we need the associated density matrix $\rho_{k}=\left|\psi_{k}\right\rangle\left\langle\psi_{k}\right|$, whose entries in the coordinate basis are

$$
\rho_{k}\left(x, y ; x^{\prime}, y^{\prime}\right)=\overline{\psi_{k}(x, y)} \psi_{k}\left(x^{\prime}, y^{\prime}\right)
$$

Now we have to trace out one of the degrees of freedom, say $y$. This is equivalent to tracing out the degrees of freedom of the particle in the gauge field theory. Then we obtain the reduced density matrix $\varrho_{k}$ associated with the gauge field $A_{\mu}$,

$$
\varrho_{k}\left(x, x^{\prime}\right)=\int_{0}^{1} \mathrm{~d} y \rho_{k}\left(x, y ; x^{\prime}, y\right) .
$$

Finally, the (von Neumann) entanglement entropy is defined as

$$
S_{k}=-\operatorname{Tr}\left(\varrho_{\mathrm{k}} \log \varrho_{\mathrm{k}}\right) .
$$

If we had chosen to trace out the degree of freedom $x$ that corresponds to the gauge field, the reduced density matrix would be associated with the charged particle. Nevertheless, the resulting entanglement entropy does not depend on which reduced density matrix we consider. 


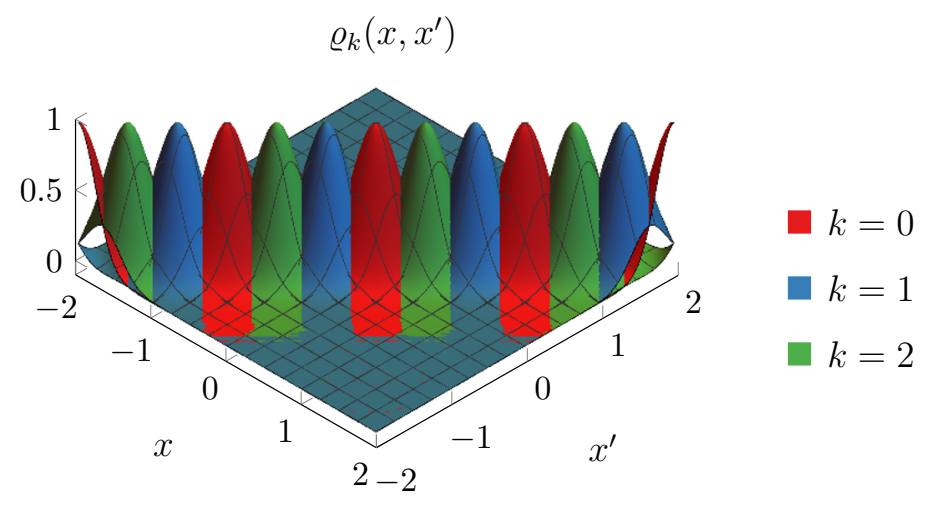

(a)
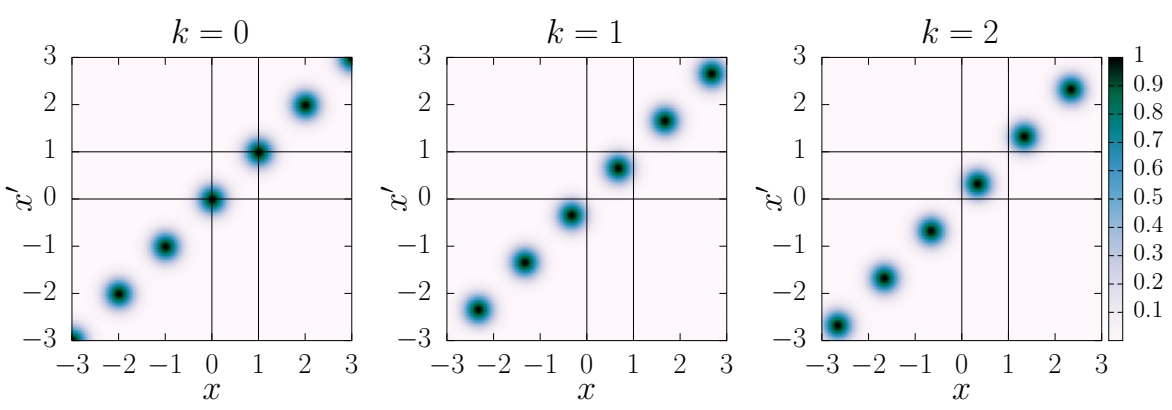

(b)

Figure 1. In (a), we represent the reduced density matrix $\varrho_{k}\left(x, x^{\prime}\right)$ obtained in (3.4) for $k=0,1,2$ with $\theta=3$. In (b), we represent the distribution of these three reduced density matrices. Note that, since the particle moves on a torus, the coordinates $x, x^{\prime}$ are restricted to the interval $[0,1)$, as the lines in (b) indicate.

Inserting the explicit expression of the wave function $\psi_{k}(x, y)$, see (2.23) and (2.27), we find that the reduced density matrix (3.2) is of the form

$$
\varrho_{k}\left(x, x^{\prime}\right)=\sum_{n \in \mathbb{Z}} \varphi_{n k}(x) \varphi_{n k}\left(x^{\prime}\right)=\mathcal{N}^{2} \sum_{n \in \mathbb{Z}} \mathrm{e}^{-\pi \theta(x+n+k / \theta)^{2}} \mathrm{e}^{-\pi \theta\left(x^{\prime}+n+k / \theta\right)^{2}},
$$

or, in terms of the $\vartheta$ function,

$$
\varrho_{k}\left(x, x^{\prime}\right)=\mathcal{N}^{2} \mathrm{e}^{-\pi \theta\left(x^{2}+x^{\prime 2}+2(k / \theta)^{2}\right)-2 \pi k\left(x+x^{\prime}\right)} \vartheta_{3}\left(\mathrm{i} \theta\left(x+x^{\prime}+2 k / \theta\right) \mid \mathrm{i} 2 \theta\right) .
$$

Now the direct way to obtain the entanglement entropy would be to compute the eigenvalues of $\varrho_{k}$ and then plug them in (3.3). However, this is in principle a difficult task that we shall bypass approximating $\varrho_{k}$ in two different ways.

First, observe that $\varrho_{k}\left(x, x^{\prime}\right)$ is made of peaks localized along the line $x^{\prime}=x$, as figure 1 (a) illustrates for $\theta=3$ and the three possible values for $k$. In figure 1 (b) we represent separately $\varrho_{k}\left(x, x^{\prime}\right)$ for each value of $k$ delimiting the interval $[0,1)$, which is the domain where the variables $x, x^{\prime}$ are defined. Observe that as $k$ grows the peaks of $\varrho_{k}\left(x, x^{\prime}\right)$ move down along the line $x^{\prime}=x$. Each peak of $\varrho_{k}\left(x, x^{\prime}\right)$ comes from one of the modes 

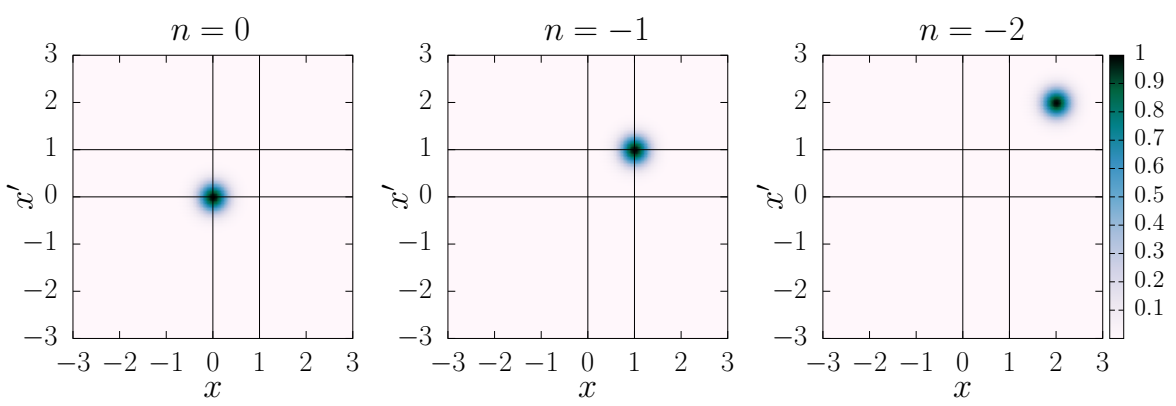

(a)
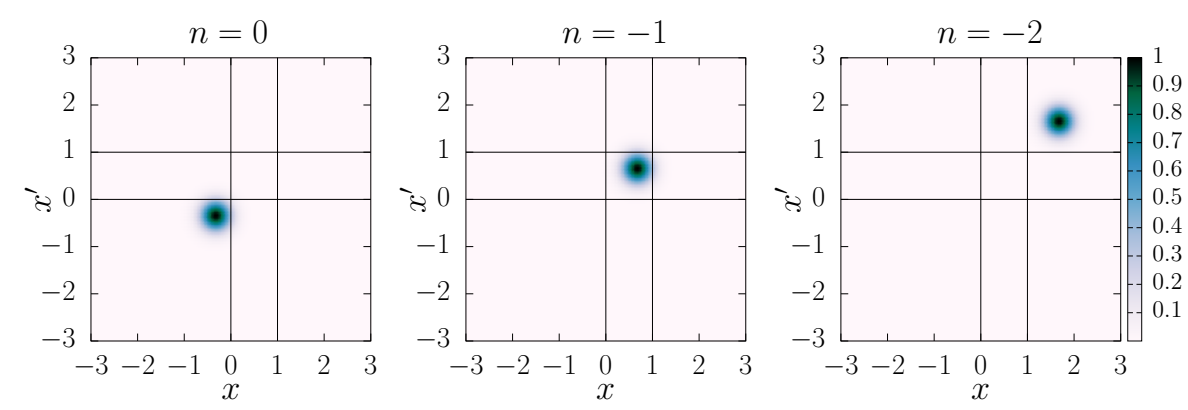

(b)
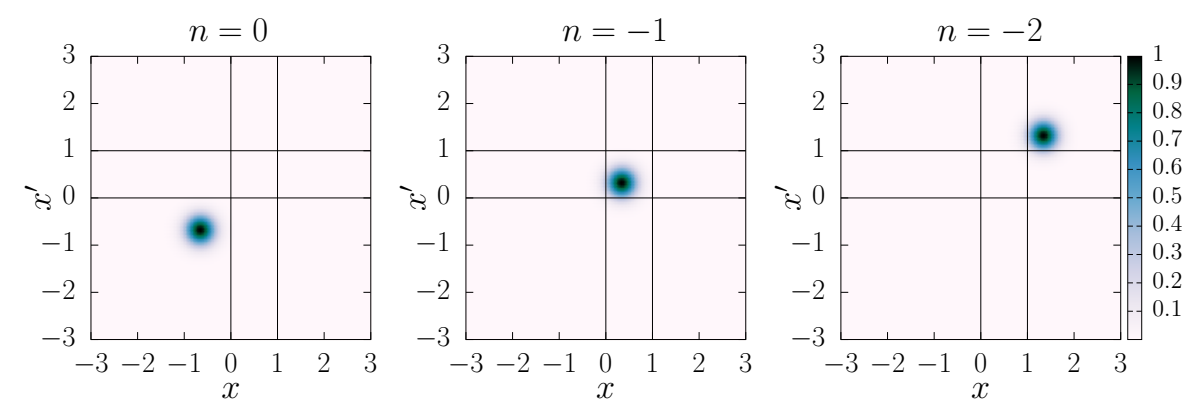

(c)

Figure 2. Contribution of the modes $n=0,-1,-2$ to the reduced density matrix $\varrho_{k}\left(x, x^{\prime}\right)$, see eq. (3.4), for $\theta=3$ and (a) $k=0$, (b) $k=1$, (c) $k=2$.

in the sum (3.4). Therefore, only the modes that correspond to a peak inside the square $[0,1) \times[0,1)$ contribute to $\varrho_{k}\left(x, x^{\prime}\right)$. For example, in figure 2 we can see that for $\theta=3$ the only modes that contribute are $n=-2,-1,0$.

In fact, for any value of $k$ and $\theta$, one can see that only two $n$ modes are significant in the interval $x \in[0,1)$ so that we can neglect the rest of them in the calculation of the reduced density matrix. Thus, we can treat the latter as that of a two-level system.

For $k / \theta<1 / 2$, only the peaks corresponding to the modes $n=0$ and $n=-1$ are relevant in the interval $[0,1)$. Therefore, the reduced density matrix can be approximated as

$$
\varrho_{k}\left(x, x^{\prime}\right) \approx \varphi_{0 k}(x) \varphi_{0 k}\left(x^{\prime}\right)+\varphi_{-1 k}(x) \varphi_{-1 k}\left(x^{\prime}\right) .
$$


If $k / \theta>1 / 2$, then the non-neglectable peaks in the interval $[0,1)$ correspond to the modes $n=-1$ and $n=-2$ and

$$
\varrho_{k}\left(x, x^{\prime}\right) \approx \varphi_{-1 k}(x) \varphi_{-1 k}\left(x^{\prime}\right)+\varphi_{-2 k}(x) \varphi_{-2 k}\left(x^{\prime}\right) .
$$

In the case $k / \theta=1 / 2$, only the peak with $n=-1$, that is at $x=1 / 2$, gives a significant contribution. Thus

$$
\varrho_{k}\left(x, x^{\prime}\right) \approx \varphi_{-1 k}(x) \varphi_{-1 k}\left(x^{\prime}\right) .
$$

These approximations may be written in terms of normalised functions $u_{n k}(x)$. To do so, we define $p_{n k}$,

$$
p_{n k}=\int_{0}^{1} \mathrm{~d} x\left|\varphi_{n k}(x)\right|^{2}=\frac{\operatorname{erf}(\sqrt{2 \pi \theta}(k / \theta+n+1))-\operatorname{erf}(\sqrt{2 \pi \theta}(k / \theta+n))}{2 \sqrt{2 \theta}},
$$

where $\operatorname{erf}(z)$ denotes the error function. In terms of $p_{n k}$ we can write the approximations as:

- For $k / \theta<1 / 2$,

$$
\varrho_{k}\left(x, x^{\prime}\right) \approx \lambda_{0 k} u_{0 k}(x) u_{0 k}\left(x^{\prime}\right)+\lambda_{-1 k} u_{-1 k}(x) u_{-1 k}\left(x^{\prime}\right)
$$

where

$$
\lambda_{n k}=\frac{p_{n k}}{p_{0 k}+p_{-1 k}} \quad \text { and } \quad u_{n k}(x)=\frac{1}{\sqrt{\lambda_{n k}}} \varphi_{n k}(x) .
$$

- For $k / \theta>1 / 2$,

$$
\varrho_{k}\left(x, x^{\prime}\right) \approx \lambda_{-1 k}^{\prime} u_{-1 k}^{\prime}(x) u_{-1 k}^{\prime}\left(x^{\prime}\right)+\lambda_{-2 k}^{\prime} u_{-2 k}^{\prime}(x) u_{-2 k}^{\prime}\left(x^{\prime}\right)
$$

where

$$
\lambda_{n k}^{\prime}=\frac{p_{n k}}{p_{-1 k}+p_{-2 k}} \quad \text { and } \quad u_{n k}^{\prime}(x)=\frac{1}{\sqrt{\lambda_{n k}^{\prime}}} \varphi_{n k}(x) .
$$

- For $k / \theta=1 / 2$,

$$
\varrho_{k}\left(x, x^{\prime}\right) \approx u_{-1 k}(x) u_{-1 k}\left(x^{\prime}\right) .
$$

Note that it follows from the definitions of $\lambda_{k n}$ and $\lambda_{k n}^{\prime}$ that $\lambda_{-1 k}=1-\lambda_{0 k}$ and $\lambda_{-2 k}^{\prime}=1-\lambda_{-1 k}^{\prime}$. Therefore, the entanglement entropy can be expressed as

$$
S_{k} \approx \begin{cases}-\lambda_{0 k} \log \lambda_{0 k}-\left(1-\lambda_{0 k}\right) \log \left(1-\lambda_{0 k}\right), & \text { if } k / \theta<1 / 2, \\ -\lambda_{-1 k} \log \lambda_{-1 k}-\left(1-\lambda_{-1 k}\right) \log \left(1-\lambda_{-1 k}\right), & \text { if } k / \theta>1 / 2, \\ 0, & \text { if } k / \theta=1 / 2 .\end{cases}
$$

The latter case only happens when $\theta$ is an even number.

Using the identity between the error function and the confluent hypergeometric function of the first kind $M(a, b, z)$ (see, e.g., eq. 13.6.7 in [52]),

$$
\operatorname{erf}(z)=\frac{2 z}{\sqrt{\pi}} M\left(\frac{1}{2}, \frac{3}{2},-z^{2}\right)
$$


we have

$$
p_{n k}=\left(\chi_{k}+n+1\right) \mathrm{M}\left(-2 \pi \theta\left(\chi_{k}+1+n\right)^{2}\right)-\left(\chi_{k}+n\right) \mathrm{M}\left(-2 \pi \theta\left(\chi_{k}+n\right)^{2}\right),
$$

where we have introduced the notation $\mathrm{M}(z) \equiv M(1 / 2,3 / 2, z)$ and $\chi_{k}=k / \theta$. Hence we find that

$$
\lambda_{0 k}=\frac{\left(\chi_{k}+1\right) \mathrm{M}\left(-2 \pi \theta\left(\chi_{k}+1\right)^{2}\right)-\chi_{k} \mathrm{M}\left(-2 \pi \theta \chi_{k}^{2}\right)}{\left(\chi_{k}+1\right) \mathrm{M}\left(-2 \pi \theta\left(\chi_{k}+1\right)^{2}\right)-\left(\chi_{k}-1\right) \mathrm{M}\left(-2 \pi \theta\left(\chi_{k}-1\right)^{2}\right)},
$$

and

$$
\lambda_{-1 k}^{\prime}=\frac{\chi_{k} \mathrm{M}\left(-2 \pi \theta \chi_{k}^{2}\right)-\left(\chi_{k}-1\right) \mathrm{M}\left(-2 \pi \theta\left(\chi_{k}-1\right)^{2}\right)}{\chi_{k} \mathrm{M}\left(-2 \pi \theta\left(\chi_{k}\right)^{2}\right)-\left(\chi_{k}-2\right) \mathrm{M}\left(-2 \pi \theta\left(\chi_{k}-2\right)^{2}\right)} .
$$

From these expressions it is clear that the entanglement entropies for $k / \theta<1 / 2$ and for $k / \theta>1 / 2$ are related by the transformation $k / \theta \mapsto 1-k / \theta$.

Let us check numerically the accuracy of the above results. This is done by expanding the functions $\varphi_{n k}(x)$ in Fourier modes,

$$
\varphi_{n k}(x)=\sum_{p \in \mathbb{Z}} \tilde{\varphi}_{n k}(p) \mathrm{e}^{2 \pi \mathrm{i} p x} .
$$

In the basis of Fourier modes the entries of the reduced density matrix (3.2) are given by

$$
\tilde{\varrho}_{k}\left(p, p^{\prime}\right)=\int \mathrm{d} x \mathrm{~d} x^{\prime} \varrho_{k}\left(x, x^{\prime}\right) \mathrm{e}^{2 \pi \mathrm{i} p x} \mathrm{e}^{-2 \pi \mathrm{i} p^{\prime} x^{\prime}}, \quad p, p^{\prime} \in \mathbb{Z} .
$$

In order to compute numerically the entanglement entropy we truncate the matrix $\left(\tilde{\varrho_{k}}\left(p, p^{\prime}\right)\right)$ restricting the indices $p, p^{\prime} \in \mathbb{Z}$ to the interval $-N \leq p, p^{\prime} \leq N$. Then we calculate the eigenvalues of this sub-matrix and we plug them in the expression of the entanglement entropy (3.3).

The value obtained numerically for the entanglement entropy should converge to that predicted by the expression (3.5) as we increase the cut-off $N$. In figure 3 we compare the results for a given $\theta$ and $k$ varying from 0 to $\theta-1$. As we can see the results agree for $N$ large enough. Notice also that the entanglement entropy varies with $k$. This means that there is an ambiguity associated to the entanglement entropy of the ground state.

It is also interesting to analyse how the entanglement entropy behaves as a function of $\theta$ for fixed $k$. Recall that $\theta$ is proportional to the electric charge of the particle (we have set $q=-2 \pi \theta$ ). In figure 4 , we plot $S_{k}$ in terms of $\theta$ for several fixed values of $k$ using the analytical approximation (3.5). The initial point of the curve for each $k$ corresponds to $\theta=k+1$. Observe that, due to the symmetry $k / \theta \mapsto 1-k / \theta$, the initial points of all the curves with $k>1$ also belong to the curve for $k=1$. As $\theta$ increases, $S_{k}$ decreases until $\theta=2 k$, where it vanishes. From this point, $S_{k}$ increases tending to $\log 2$ when $\theta \rightarrow \infty$ (infinite transverse magnetic flux in the associated Landau model). Note that as $k$ is larger, the entropy saturates more slowly to the asymptotic value $\log 2$. We can conclude that there is an upper bound for the entanglement entropy in the states $\left|\psi_{k}\right\rangle$, which is exactly that of a maximally entangled two-level quantum system. 


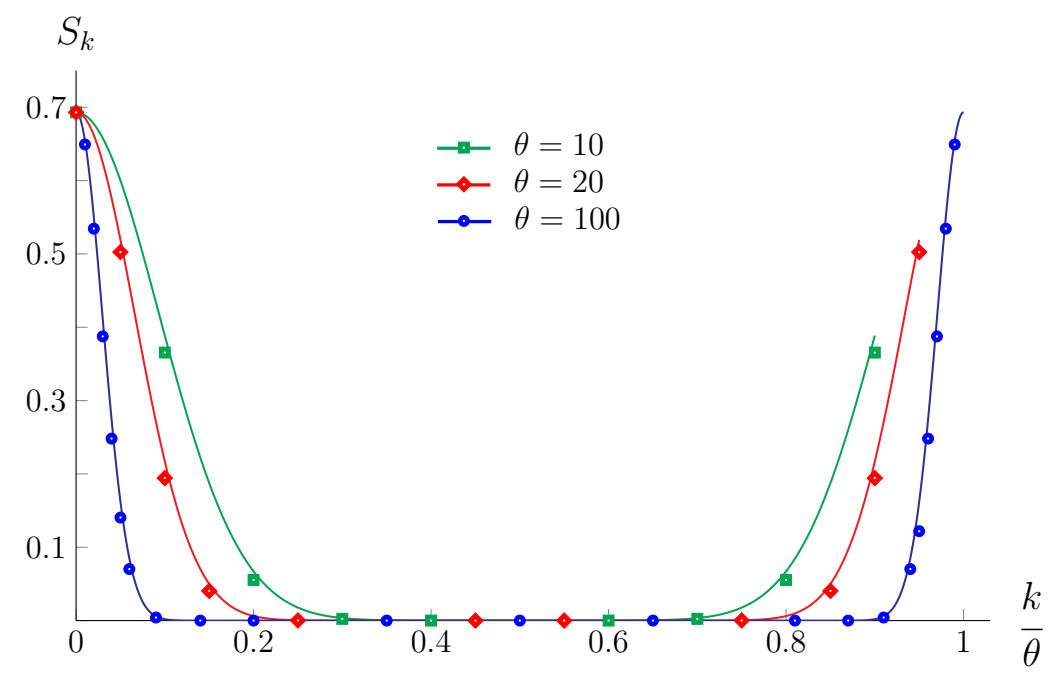

Figure 3. Entanglement entropy as a function of the ratio $k / \theta$ for several fixed values of $\theta$ and varying $k$. The solid lines represent the analytical approximation obtained in eq. (3.5). The dots have been obtained numerically from the matrix (3.6), taking as cut-off $N=100$.

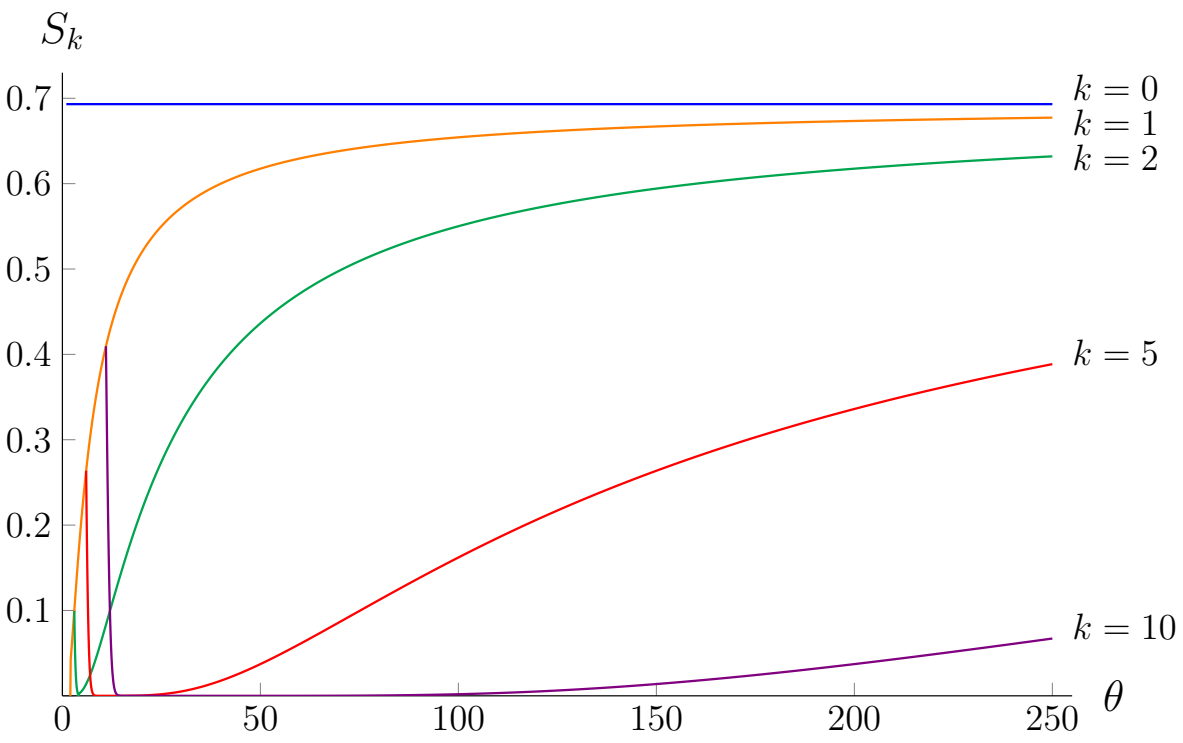

Figure 4. Entanglement entropy as a function of $\theta$ for some fixed values of $k$ using the analytical approximation found in (3.5). 
Another interesting case is $\theta=0$. It corresponds to a particle with zero electric charge (zero transverse magnetic flux in the Landau model). For $\theta=0$, the analytical approximation (3.5) is not well defined. Nevertheless, since the particle and the gauge field are decoupled, the degrees of freedom of the Landau model, $x$ and $y$, are separable; that is, the wave function of the ground state, that now is not degenerate, can be factorized in the form $\psi(x, y)=X(x) Y(y)$. This implies that the entanglement entropy is zero for $\theta=0$.

\subsection{Entanglement entropy for a $V_{\theta}$-invariant state}

We have just seen that the entanglement entropy changes inside the degeneracy space of the ground state; that is, according to eq. (2.26), it varies under the discrete translations in the $x$ direction of the torus defined by $V_{\theta}$. However, there is a state which is invariant under $V_{\theta}$ : the linear combination of the states $\left\{\left|\psi_{k}\right\rangle\right\}_{k=0}^{\theta-1}$ that span the ground state degeneracy subspace

$$
\left|\psi_{\theta}\right\rangle=\frac{1}{\sqrt{\theta}} \sum_{k=0}^{\theta-1}\left|\psi_{k}\right\rangle .
$$

In the coordinate basis, its density matrix $\rho_{\theta}=\left|\psi_{\theta}\right\rangle\left\langle\psi_{\theta}\right|$ reads

$$
\rho_{\theta}\left(x, y ; x^{\prime}, y^{\prime}\right)=\frac{1}{\theta} \sum_{k, k^{\prime}=0}^{\theta-1} \overline{\psi_{k}(x, y)} \psi_{k^{\prime}}\left(x^{\prime}, y^{\prime}\right) .
$$

In order to calculate the entanglement entropy of $\left|\psi_{\theta}\right\rangle$, we can take the partial trace in (3.8) with respect to either $x$ or $y$. It is more convenient to take it with respect to the former. The corresponding reduced density matrix,

$$
\sigma_{\theta}\left(y, y^{\prime}\right)=\int_{0}^{1} \mathrm{~d} x \rho_{\theta}\left(x, y ; x, y^{\prime}\right)
$$

can be expressed in the form

$$
\sigma_{\theta}\left(y, y^{\prime}\right)=\sum_{p, p^{\prime} \in \mathbb{Z}} \tilde{\sigma}_{\theta}\left(p, p^{\prime}\right) \mathrm{e}^{-2 \pi \mathrm{i} p y} \mathrm{e}^{2 \pi \mathrm{i} p^{\prime} y^{\prime}},
$$

with

$$
\tilde{\sigma}_{\theta}\left(p, p^{\prime}\right)=\mathcal{N}^{2} \frac{\mathrm{e}^{-\pi\left(p-p^{\prime}\right)^{2} /(2 \theta)}}{2 \theta \sqrt{2 \theta}}\left(\operatorname{erf}\left(\sqrt{\frac{\pi}{2 \theta}}\left(2 \theta+p+p^{\prime}\right)\right)-\operatorname{erf}\left(\sqrt{\frac{\pi}{2 \theta}}\left(p+p^{\prime}\right)\right)\right) .
$$

Observe that the elements $\tilde{\sigma}_{\theta}\left(p, p^{\prime}\right), p, p^{\prime} \in \mathbb{Z}$, form an infinite matrix which represents $\sigma_{\theta}$ in the momentum space. As we have done before, we can calculate the entanglement entropy $S_{\theta}$ of $\left|\psi_{\theta}\right\rangle$ from the spectrum of this matrix truncated for $|p|,\left|p^{\prime}\right|$ large enough. In figure 5, we have computed numerically $S_{\theta}$ varying $\theta$ using this method. From this plot, we can conclude that

$$
S_{\theta} \sim \frac{1}{2} \log \theta+\alpha+\frac{\beta}{\sqrt{\theta}}, \quad \text { for } \quad \theta \gg 1
$$




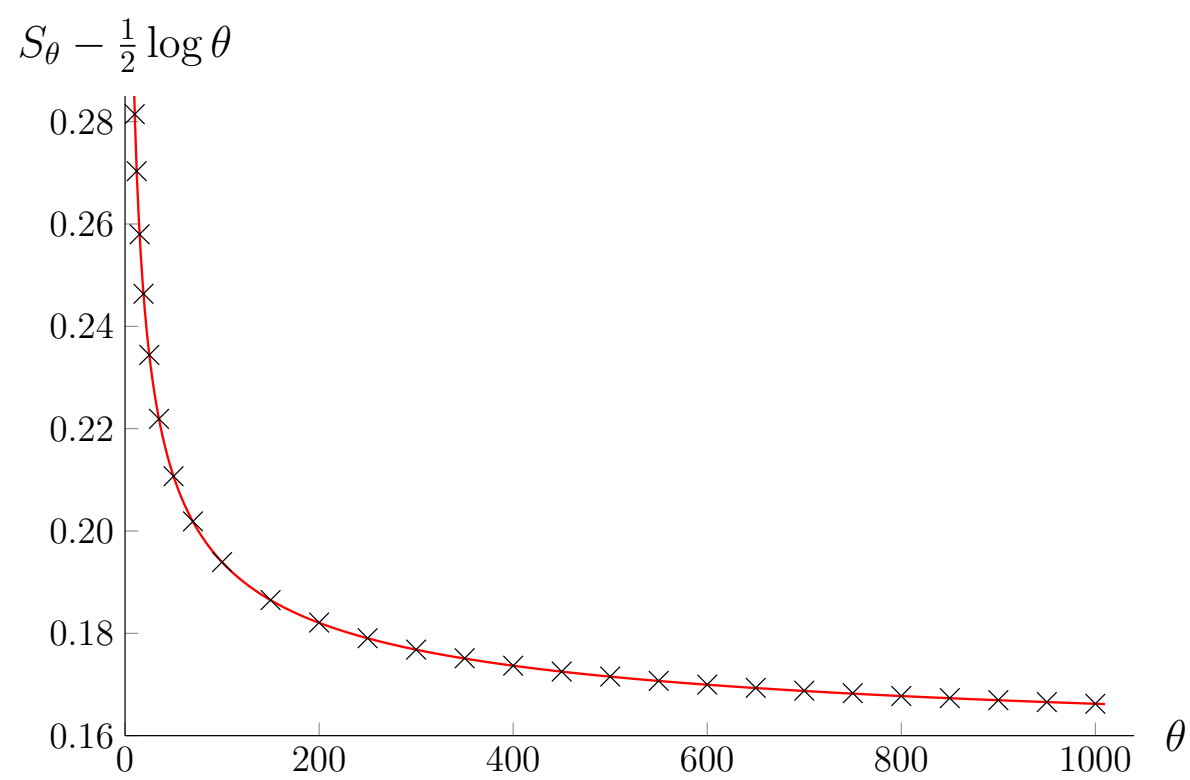

Figure 5. Entanglement entropy of the $V_{\theta}$-invariant state (3.8) substracting the term $1 / 2 \log \theta$ as a function of $\theta$. The crosses correspond to the values obtained for $S_{\theta}$ by diagonalizing numerically the reduced density matrix (3.9), restricting the indices to $-1100 \leq p, p^{\prime} \leq 1100$. The solid line is the curve $\alpha+\beta / \sqrt{\theta}$ fitted to the numerical points in the interval $\theta \in[500,1000]$; we obtain $\alpha=0.15343$ and $\beta=0.404832$.

The value of the coefficients $\alpha, \beta$, see the caption of figure 5 , can be determined from the fit of (3.10) to the numerical data. Note in this figure that the fitted curve $\alpha+\beta / \sqrt{\theta}$ is very close to the numerical data. This is specially remarkable if we take into account that the fit was performed for values of $\theta$ between 500 and 1000 and then the curve is plotted from $\theta=10$.

Therefore, contrary to the states $\left|\psi_{k}\right\rangle$, eigenfunctions of the translations $V_{y}(a)$ in the $y$ direction of the torus, whose entanglement entropy tends to $\log 2$ in the limit $\theta \rightarrow \infty$ (see figure 4), the entanglement entropy of the state $\left|\psi_{\theta}\right\rangle$, invariant under the translations $V_{x}(a)$ in the $x$ direction, diverges logarithmically with the transverse magnetic flux/charge of the particle.

It is worth commenting that another state invariant under $V_{\theta}$ is the mixed state described by the density matrix

$$
\rho_{\mathrm{M}}=\frac{1}{\theta} \sum_{k=0}^{\theta-1}\left|\psi_{k}\right\rangle\left\langle\psi_{k}\right|
$$

or, in the coordinate basis,

$$
\rho_{\mathrm{M}}\left(x, y ; x^{\prime}, y^{\prime}\right)=\frac{1}{\theta} \sum_{k=0}^{\theta-1} \rho_{k}\left(x, y ; x^{\prime}, y^{\prime}\right) .
$$


It corresponds to the equiprobable classical ensemble of the states $\left\{\left|\psi_{k}\right\rangle\right\}_{k=0}^{\theta-1}$. It is easy to see that the partial traces of $\rho_{\mathrm{M}}$ and $\rho_{\theta}$ with respect to $y$,

$$
\varrho_{\mathrm{M}}\left(x, x^{\prime}\right)=\int_{0}^{1} \mathrm{~d} y \rho_{\mathrm{M}}\left(x, y ; x^{\prime}, y\right), \quad \varrho_{\theta}\left(x, x^{\prime}\right)=\int_{0}^{1} \mathrm{~d} y \rho_{\theta}\left(x, y ; x^{\prime}, y\right)
$$

lead to the same reduced density matrix

$$
\varrho_{\theta}\left(x, x^{\prime}\right)=\varrho_{\mathrm{M}}\left(x, x^{\prime}\right)=\frac{1}{\theta} \sum_{k=0}^{\theta-1} \varrho_{k}\left(x, x^{\prime}\right) .
$$

This means that, from the perspective of the field theory problem, the gauge field cannot distinguish if the whole system is in the linear combination or in the statistical ensemble of the states $\left\{\left|\psi_{k}\right\rangle\right\}_{k=0}^{\theta-1}$. The same is not true for the particle. The partial trace of (3.11) with respect to $x$,

$$
\sigma_{\mathrm{M}}\left(y, y^{\prime}\right)=\int_{0}^{1} \mathrm{~d} x \rho_{\mathrm{M}}\left(x, y ; x, y^{\prime}\right),
$$

can be written in the momentum space as

$$
\tilde{\sigma}_{\mathrm{M}}\left(p, p^{\prime}\right)=\delta_{p, p^{\prime}}^{\bmod \theta} \tilde{\sigma}_{\theta}\left(p, p^{\prime}\right)
$$

where $\delta_{p, p^{\prime}}^{\bmod \theta}$ is 1 if $p=p^{\prime}(\bmod \theta)$ and 0 otherwise. One can numerically check that the entropies obtained from $\sigma_{\mathrm{M}}$ and $\sigma_{\theta}$ are indeed different.

\section{Conclusions}

In this work, we studied the ground state entanglement entropy between an electromagnetic field and a charged non-relativistic particle on a space-time cylinder. In order to compute this entropy, we resorted to the fact that a Yang-Mills field theory defined on a space-time cylinder can be mapped to the problem of a free quantum particle moving on the gauge group manifold. In our case, we considered an electromagnetic field, for which the gauge group is $\mathrm{U}(1)$, and therefore the corresponding manifold is the unit circle. Since here the gauge field is coupled to a non-relativistic particle, the associated quantum mechanical problem is a particle moving on a torus with a transverse magnetic field: the Landau model on a torus. The two degrees of freedom of the particle on the torus correspond respectively to the gauge field and to the non-relativistic particle in the field theory problem.

Therefore, the computation of the entanglement entropy between the electromagnetic field and the non-relativistic particle was reduced to taking the partial trace of one of the degrees of freedom in the wave function of the particle on the torus and computing the entropy from the corresponding reduced density matrix. Since the ground state of the Landau model is degenerate, we analysed the entanglement entropy in the degeneracy subspace. We performed this analysis treating the reduced density matrix of the states that generate this subspace as that of a two-level system. We obtained an approximate analytical expression for their entanglement entropy which was checked numerically. In particular, we found that, when the electromagnetic field and the particle are decoupled, 
the entanglement entropy is zero while, when the particle's charge goes to infinity, the entanglement entropy tends to $\log 2$.

The symmetry behind the degeneracy of the ground state is the group of translations $\mathbb{Z}_{\theta}$ in the $y$ direction of the torus. The translational symmetry is anomalously broken due to the boundary conditions of the Landau Hamiltonian to the discrete subgroup $\mathbb{Z}_{\theta} \times \mathbb{Z}_{\theta}$, where $\theta$ is the electric charge of the particle in the field theory/the transverse magnetic flux in the Landau model, which is quantized. We also studied the entanglement of the state invariant under the $\mathbb{Z}_{\theta}$ translations in the $x$ direction of the torus. This state can be constructed from the equiprobable linear combination of the states that generate the ground state degeneracy subspace. In this case, the entanglement entropy does not saturate to a finite value when the particle's charge goes to infinity, but it scales logarithmically with the charge.

The natural continuation of this work is to take a non-Abelian Yang-Mills theory instead of an Abelian one and study how the results obtained here generalise to the $\mathrm{SU}(N)$ gauge group. In particular, the YM theory would be mapped to a particle moving along a different gauge group manifold. For example, for SU(2) we would have a particle moving along $S^{3}$. Solving its dynamics would then mean working with a non-trivial set of Wong's equations [53]. Another interesting aspect to analyse is the evolution of the entanglement between the matter and the gauge sectors after a quantum quench $[54,55]$. This could be done by preparing the system in the ground state in which the gauge field and the particle are decoupled and then suddenly turning on the interaction term, for example. Indeed, the non-equilibrium dynamics of a $1+1$ dimensional $\mathrm{U}(1)$ gauge theory coupled either to fermions [56] or to bosons [57] has recently been investigated and it was observed that the system may not thermalize. We plan to tackle these problems in the future.

\section{Acknowledgments}

We thank A. Melikyan, A. Pinzul and D. Trancanelli for valuable comments on the manuscript. FA acknowlegdes financial support from the Brazilian ministries MEC and MCTIC. MT acknowledges the support of the Conselho Nacional de Desenvolvimento Cientifico e Tecnologico (CNPq). We thank the anonymous referee of JHEP for the interesting comments and questions which have allowed us to improve this work.

\section{A Green's function}

We want to solve $\partial_{s}^{2} G\left(s, s^{\prime}\right)=\delta\left(s-s^{\prime}\right)$ in the domain $s, s^{\prime} \in[0,2 \pi R)$ with periodic boundary conditions $G\left(s+2 \pi R, s^{\prime}\right)=G\left(s, s^{\prime}+2 \pi R\right)=G\left(s, s^{\prime}\right)$. Since the system is translationally invariant, $G\left(s, s^{\prime}\right) \equiv G\left(s-s^{\prime}\right)$. Therefore, we can rewrite the problem in terms of the variable $\mathbf{s}=s-s^{\prime}$ as $\partial_{\mathbf{s}}^{2} G(\mathbf{s})=\delta(\mathbf{s})$ with boundary condition $G(\mathbf{s})=G(\mathbf{s}+2 \pi R)$. In the end, we will just need to replace $\mathrm{s}$ by $s-s^{\prime}$.

In order to solve $\partial_{\mathbf{s}}^{2} G(\mathbf{s})=\delta(\mathbf{s})$, we express $G(\mathbf{s})$ and $\delta(\mathbf{s})$ in terms of their Fourier series,

$$
G(\mathbf{s})=a_{0}+\sum_{n \neq 0} a_{n} \mathrm{e}^{\mathrm{i} n \mathbf{s} / R}, \quad \text { and } \quad \delta(\mathbf{s})=\frac{1}{2 \pi R}+\frac{1}{2 \pi R} \sum_{n \neq 0} \mathrm{e}^{\mathrm{i} n \mathbf{s} / R} .
$$


After plugging them in the differential equation, we obtain

$$
\sum_{n \neq 0}\left(\frac{\mathrm{i} n}{R}\right)^{2} a_{n} \mathrm{e}^{\mathrm{i} n \mathrm{~s} / R} \stackrel{!}{=} \frac{1}{2 \pi R}+\frac{1}{2 \pi R} \sum_{n \neq 0} \mathrm{e}^{\mathrm{i} n \mathrm{~s} / R} .
$$

Note that the presence of a zero mode on the right-hand side is problematic. To circumvent this issue, we remove it for now. We will discuss the validity of this later. Going back to the differential equation, we should now solve

$$
\sum_{n \neq 0}\left(\frac{\mathrm{i} n}{R}\right)^{2} a_{n} \mathrm{e}^{\mathrm{i} n \mathrm{~s} / R}=\frac{1}{2 \pi R} \sum_{n \neq 0} \mathrm{e}^{\mathrm{i} n \mathrm{~s} / R},
$$

which gives $a_{n}=-\frac{R}{2 \pi n^{2}}$. Therefore,

$$
G(\mathbf{s})=-\sum_{n \neq 0} \frac{R}{2 \pi n^{2}} \mathrm{e}^{\mathrm{i} n \mathrm{~s} / R}
$$

Now the problem boils down to computing this infinite sum. To do so, we note that

$$
\begin{aligned}
G(\mathbf{s})=-\sum_{n=-\infty}^{-1} \frac{R}{2 \pi n^{2}} \mathrm{e}^{\mathrm{i} n \mathbf{s} / R}-\sum_{n=1}^{\infty} \frac{R}{2 \pi n^{2}} \mathrm{e}^{\mathrm{i} n \mathbf{s} / R} & =-\frac{R}{2 \pi} \sum_{n=1}^{\infty}\left(\frac{\mathrm{e}^{-\mathrm{i} n \mathbf{s} / R}}{n^{2}}+\frac{\mathrm{e}^{\mathrm{i} n \mathbf{s} / R}}{n^{2}}\right) \\
& =-\frac{R}{2 \pi}\left(\operatorname{Li}_{2}\left(\mathrm{e}^{-\mathrm{i} / R}\right)+\operatorname{Li}_{2}\left(\mathrm{e}^{\mathrm{i} \mathbf{s} / R}\right)\right),
\end{aligned}
$$

where $\mathrm{Li}_{2}(z)$ is the dilogarithm function. Using the identity (see, e.g., eq. 25.12.4 in [52]),

$$
\operatorname{Li}_{2}(z)+\operatorname{Li}_{2}\left(\frac{1}{z}\right)=-\frac{\pi^{2}}{6}-\frac{1}{2}(\ln (-z))^{2},
$$

which for the case $z=\mathrm{e}^{\mathrm{i} \phi}, \phi \in[-2 \pi, 2 \pi]$, reads

$$
\mathrm{Li}_{2}\left(\mathrm{e}^{\mathrm{i} \phi}\right)+\mathrm{Li}_{2}\left(\mathrm{e}^{-\mathrm{i} \phi}\right)=-\frac{\pi^{2}}{6}+\frac{1}{2}(|\phi|-\pi)^{2},
$$

we finally obtain

$$
G(\mathrm{~s})=a_{0}-\frac{\mathrm{s}^{2}}{4 \pi R}+\frac{|\mathbf{s}|}{2}-\frac{\pi R}{6} .
$$

with $\mathbf{s} \in[-2 \pi R, 2 \pi R]$. The zero mode $a_{0}$ is irrelevant and we take it to be zero.

In terms of $s, s^{\prime} \in \mathbb{R}$, we have

$$
G\left(s, s^{\prime}\right)=-\frac{\left([s]-\left[s^{\prime}\right]\right)^{2}}{4 \pi R}+\frac{\left|[s]-\left[s^{\prime}\right]\right|}{2}-\frac{\pi R}{6},
$$

satisfying

$$
\partial_{s}^{2} G\left(s, s^{\prime}\right)=\delta\left(s-s^{\prime}\right)-\frac{1}{2 \pi R} .
$$


Finally, we mention that removing the zero mode of the Dirac delta does not compromise the result. In fact, if we act with $\partial_{s}^{2}$ on (2.5), we obtain

$$
\begin{aligned}
\partial_{s}^{2} A_{0}(s)=\int \mathrm{d} s^{\prime} \partial_{s}^{2} G\left(s, s^{\prime}\right) \rho\left(s^{\prime}\right) & =\int \mathrm{d} s^{\prime}\left(\delta\left(s-s^{\prime}\right)-\frac{1}{2 \pi R}\right) \rho\left(s^{\prime}\right) \\
& =\rho(s)-\frac{1}{2 \pi R} \int \mathrm{d} s^{\prime} \rho\left(s^{\prime}\right) .
\end{aligned}
$$

Since the electric field satisfies periodic boundary conditions, the last term vanishes,

$$
\int \mathrm{d} s^{\prime} \rho\left(s^{\prime}\right)=\int \mathrm{d} s^{\prime} \partial_{s^{\prime}} E\left(s^{\prime}\right)=0 .
$$

Thus $G\left(s, s^{\prime}\right)$ indeed solves the Gauss law constraint.

Open Access. This article is distributed under the terms of the Creative Commons Attribution License (CC-BY 4.0), which permits any use, distribution and reproduction in any medium, provided the original author(s) and source are credited.

\section{References}

[1] E. Schrödinger, Discussion of probability relations between separated systems, Math. Proc. Cambridge Philos. Soc. 31 (1935) 555.

[2] M.A. Nielsen and I.L. Chuang, Quantum computation and quantum information, Cambridge Univ. Press, Cambridge, U.K. (2000).

[3] R. Horodecki, P. Horodecki, M. Horodecki and K. Horodecki, Quantum entanglement, Rev. Mod. Phys. 81 (2009) 865 [quant-ph/0702225] [INSPIRE].

[4] L. Amico, R. Fazio, A. Osterloh and V. Vedral, Entanglement in many-body systems, Rev. Mod. Phys. 80 (2008) 517 [quant-ph/0703044] [INSPIRE].

[5] P. Calabrese, J. Cardy and B. Doyon, Entanglement entropy in extended quantum systems, J. Phys. A 42 (2009) 500301.

[6] N. Laflorencie, Quantum entanglement in condensed matter systems, Phys. Rept. 646 (2016) 1 [arXiv: 1512.03388] [InSPIRE].

[7] S.N. Solodukhin, Entanglement entropy of black holes, Living Rev. Rel. 14 (2011) 8 [arXiv:1104.3712] [INSPIRE].

[8] C.H. Bennett, H.J. Bernstein, S. Popescu and B. Schumacher, Concentrating partial entanglement by local operations, Phys. Rev. A 53 (1996) 2046 [quant-ph/9511030] [INSPIRE].

[9] L. Bombelli, R.K. Koul, J. Lee and R.D. Sorkin, A quantum source of entropy for black holes, Phys. Rev. D 34 (1986) 373 [InSPIRE].

[10] M. Srednicki, Entropy and area, Phys. Rev. Lett. 71 (1993) 666 [hep-th/9303048] [INSPIRE].

[11] C. Holzhey, F. Larsen and F. Wilczek, Geometric and renormalized entropy in conformal field theory, Nucl. Phys. B 424 (1994) 443 [hep-th/9403108] [INSPIRE].

[12] P. Calabrese and J.L. Cardy, Entanglement entropy and quantum field theory, J. Stat. Mech. 0406 (2004) P06002 [hep-th/0405152] [INSPIRE]. 
[13] S. Ryu and T. Takayanagi, Holographic derivation of entanglement entropy from AdS/CFT, Phys. Rev. Lett. 96 (2006) 181602 [hep-th/0603001] [INSPIRE].

[14] M. Van Raamsdonk, Lectures on gravity and entanglement, in Theoretical advanced study institute in elementary particle physics: new frontiers in fields and strings, World Scientific, Singapore (2017), pg. 297 [arXiv: 1609.00026] [INSPIRE].

[15] H. Casini and M. Huerta, A finite entanglement entropy and the c-theorem, Phys. Lett. B 600 (2004) 142 [hep-th/0405111] [INSPIRE].

[16] H. Casini, C.D. Fosco and M. Huerta, Entanglement and alpha entropies for a massive Dirac field in two dimensions, J. Statist. Mech. 2005 (2005) P07007 [cond-mat/0505563] [INSPIRE].

[17] H. Casini and M. Huerta, Entanglement and alpha entropies for a massive scalar field in two dimensions, J. Statist. Mech. 2005 (2005) P12012 [cond-mat/0511014] [INSPIRE].

[18] H. Casini and M. Huerta, Entanglement entropy in free quantum field theory, J. Phys. A 42 (2009) 504007 [arXiv: 0905 . 2562] [INSPIRE].

[19] J.L. Cardy, O.A. Castro-Alvaredo and B. Doyon, Form factors of branch-point twist fields in quantum integrable models and entanglement entropy, J. Statist. Phys. 130 (2008) 129 [arXiv:0706.3384] [INSPIRE].

[20] O.A. Castro-Alvaredo and B. Doyon, Bi-partite entanglement entropy in massive 1+1-dimensional quantum field theories, J. Phys. A 42 (2009) 504006 [arXiv:0906.2946] [INSPIRE].

[21] L.A. Pando Zayas and N. Quiroz, Left-right entanglement entropy of boundary states, JHEP 01 (2015) 110 [arXiv:1407.7057] [INSPIRE].

[22] D. Das and S. Datta, Universal features of left-right entanglement entropy, Phys. Rev. Lett. 115 (2015) 131602 [arXiv:1504.02475] [INSPIRE].

[23] M. Lencses, J. Viti and G. Takács, Chiral entanglement in massive quantum field theories in $1+1$ dimensions, JHEP 01 (2019) 177 [arXiv:1811.06500] [INSPIRE].

[24] A. Prudenziati and D. Trancanelli, Replica trick and string winding, Phys. Rev. D 96 (2017) 026009 [arXiv: 1610.07618] [INSPIRE].

[25] D.N. Kabat, Black hole entropy and entropy of entanglement, Nucl. Phys. B 453 (1995) 281 [hep-th/9503016] [INSPIRE].

[26] P.V. Buividovich and M.I. Polikarpov, Entanglement entropy in gauge theories and the holographic principle for electric strings, Phys. Lett. B 670 (2008) 141 [arXiv:0806.3376] [INSPIRE].

[27] W. Donnelly, Decomposition of entanglement entropy in lattice gauge theory, Phys. Rev. D 85 (2012) 085004 [arXiv: 1109.0036] [INSPIRE].

[28] S. Ghosh, R.M. Soni and S.P. Trivedi, On the entanglement entropy for gauge theories, JHEP 09 (2015) 069 [arXiv: 1501.02593] [INSPIRE].

[29] R.M. Soni and S.P. Trivedi, Aspects of entanglement entropy for gauge theories, JHEP 01 (2016) 136 [arXiv: 1510.07455] [INSPIRE].

[30] S. Aoki, T. Iritani, M. Nozaki, T. Numasawa, N. Shiba and H. Tasaki, On the definition of entanglement entropy in lattice gauge theories, JHEP 06 (2015) 187 [arXiv:1502.04267] [INSPIRE]. 
[31] J. Lin and D. Radičević, Comments on defining entanglement entropy, arXiv:1808.05939 [INSPIRE].

[32] A. Gromov and R.A. Santos, Entanglement entropy in $2 D$ non-Abelian pure gauge theory, Phys. Lett. B 737 (2014) 60 [arXiv:1403.5035] [INSPIRE].

[33] A. Velytsky, Entanglement entropy in $d+1 \mathrm{SU}(N)$ gauge theory, Phys. Rev. D 77 (2008) 085021 [arXiv: 0801.4111] [INSPIRE].

[34] W. Donnelly, Entanglement entropy and non-Abelian gauge symmetry, Class. Quant. Grav. 31 (2014) 214003 [arXiv:1406.7304] [InSPIRE].

[35] A. Agarwal, D. Karabali and V.P. Nair, Gauge-invariant variables and entanglement entropy, Phys. Rev. D 96 (2017) 125008 [arXiv:1701.00014] [INSPIRE].

[36] H. Casini, M. Huerta and J.A. Rosabal, Remarks on entanglement entropy for gauge fields, Phys. Rev. D 89 (2014) 085012 [arXiv:1312.1183] [InSPIRE].

[37] A.P. Balachandran, T.R. Govindarajan, A.R. de Queiroz and A.F. Reyes-Lega, Entanglement and particle identity: a unifying approach, Phys. Rev. Lett. 110 (2013) 080503 [arXiv: 1303.0688] [INSPIRE].

[38] A.P. Balachandran, T.R. Govindarajan, A.R. de Queiroz and A.F. Reyes-Lega, Algebraic approach to entanglement and entropy, Phys. Rev. A 88 (2013) 022301 [arXiv:1301.1300] [INSPIRE].

[39] A.P. Balachandran, A.R. de Queiroz and S. Vaidya, Entropy of quantum states: ambiguities, Eur. Phys. J. Plus 128 (2013) 112 [arXiv:1212.1239] [INSPIRE].

[40] A.P. Balachandran, A.R. de Queiroz and S. Vaidya, Quantum entropic ambiguities: ethylene, Phys. Rev. D 88 (2013) 025001 [arXiv:1302.4924] [InSPIRE].

[41] H. Casini, M. Huerta, J.M. Magán and D. Pontello, Logarithmic coefficient of the entanglement entropy of a Maxwell field, Phys. Rev. D 101 (2020) 065020 [arXiv: 1911.00529] [INSPIRE].

[42] H. Casini, M. Huerta, J.M. Magán and D. Pontello, Entanglement entropy and superselection sectors. Part I. Global symmetries, JHEP 02 (2020) 014 [arXiv: 1905.10487] [INSPIRE].

[43] S. Aoki, N. Iizuka, K. Tamaoka and T. Yokoya, Entanglement entropy for $2 D$ gauge theories with matters, Phys. Rev. D 96 (2017) 045020 [arXiv:1705.01549] [INSPIRE].

[44] D. Makarov, Quantum entanglement of a harmonic oscillator with an electromagnetic field, Sci. Rep. 8 (2018) 8204 [arXiv:1709.04716] [INSPIRE].

[45] L.D. Landau, Diamagnetismus der Metalle (in German), Z. Phys. 64 (1930) 629.

[46] N.S. Manton, The Schwinger model and its axial anomaly, Annals Phys. 159 (1985) 220 [INSPIRE].

[47] S.G. Rajeev, Yang-Mills theory on a cylinder, Phys. Lett. B 212 (1988) 203 [InSPIRE].

[48] M. Asorey, J. Esteve and A. Pacheco, Planar rotor: the $\theta$-vacuum structure, and some approximate methods in quantum mechanics, Phys. Rev. D 27 (1983) 1852 [InSPIRE].

[49] J.G. Esteve, Anomalies in conservation laws in the Hamiltonian formalism, Phys. Rev. D 34 (1986) 674 [INSPIRE].

[50] E. Onofri, Landau levels on a torus, Int. J. Theor. Phys. 40 (2001) 537 [quant-ph/0007055] [INSPIRE]. 
[51] J.G. Esteve, Origin of the anomalies: the modified Heisenberg equation, Phys. Rev. D 66 (2002) 125013 [hep-th/0207164] [INSPIRE].

[52] F.W.J. Olver et al. eds., NIST digital library of mathematical functions, release 1.0.18, http://dlmf.nist.gov/, 27 March 2018.

[53] S.K. Wong, Field and particle equations for the classical Yang-Mills field and particles with isotopic spin, Nuovo Cim. A 65 (1970) 689 [INSPIRE].

[54] S. Ghosh, K.S. Gupta and S.C.L. Srivastava, Entanglement dynamics following a sudden quench: an exact solution, EPL 120 (2017) 50005 [arXiv: 1709.02202] [INSPIRE].

[55] S. Ghosh, K.S. Gupta and S.C.L. Srivastava, Exact relaxation dynamics and quantum information scrambling in multiply quenched harmonic chains, Phys. Rev. E 100 (2019) 012215 [arXiv: 1905.06743] [INSPIRE].

[56] M. Brenes, M. Dalmonte, M. Heyl and A. Scardicchio, Many-body localization dynamics from gauge invariance, Phys. Rev. Lett. 120 (2018) 030601 [arXiv:1706.05878] [INSPIRE].

[57] T. Chanda, J. Zakrzewski, M. Lewenstein and L. Tagliacozzo, Confinement and lack of thermalization after quenches in the bosonic Schwinger model, Phys. Rev. Lett. 124 (2020) 180602 [arXiv: 1909.12657] [INSPIRE]. 